http://jmscr.igmpublication.org/home/ ISSN (e)-2347-176x ISSN (p) 2455-0450

crossref DOI: https://dx.doi.org/10.18535/jmscr/v7i11.91

\title{
Spectrum of histopathological study of colonoscopic biopsies in a tertiary care hospital
}

\author{
Authors \\ B.Vani ${ }^{1}$, Swathi Cheruku ${ }^{2 *}$ \\ ${ }^{1,2}$ Assistant Professor, Department of Pathology, Malla Reddy Institute of Medical Sciences, Hyderabad, \\ Telangana State, India \\ *Corresponding Author \\ Dr Swathi Cheruku
}

Assistant Professor, Department of Pathology, Malla Reddy Institute of Medical Sciences, Hyderabad,

Telangana State, India

Abstract
Introduction: Colorectal cancer ranks second among the most common tumours of the world according
to the World Cancer Report of 2000. The incidence in India is about 7/1,00,000. The increasing role of
colonoscopy and polypectomy in the diagnosis and management of colorectal adenomas during the past
decade has provided the histopathologist with an abundance of valuable material for study.
Materials \& Methods: This was a prospective study carried out over a period of 26 months on all the
colonoscopic biopsy specimens received in the Department of Pathology. All the colonoscopic biopsies
received in Dept of Pathology were considered in the study. The resected specimens of large intestine
were excluded from the study. All the clinical findings and radiological diagnosis were also considered.
Results: In the present study, total of 76 cases were considered. Among them 24 cases were
inflammatory, 19 benign and 33 malignant lesions. Among the 76 patients, 45 were males and 31 females
with a male to female ratio of 1.5:1. Age group between 41 to 50 years were most commonly affected.
Conclusion: Colonoscopic examination with biopsy may be used for follow up of Inflammatory bowel
diseases and to define epithelial dysplasia in cases of chronic colitis and thus help in the early detection
of carcinoma in such cases. Hence, colonoscopic biopsy has improved the role of pathologists in the
diagnosis and management of large bowel diseases while underscoring a constant correlation with
clinicians, but a correct interpretation still proves to be an exciting diagnostic challenge for pathologists
because of small size of the specimens.
Keywords: Inflammatory bowel diseases, Papilloma, Adenocarcinoma.

\section{Introduction}

The study of colonoscopic biopsies is fascinating; among these lesions intestinal neoplasms constitute a considerable share ${ }^{(1)}$. The colon and rectum can be sites for infections, idiopathic inflammatory bowel disease (IBD), vascular disorders, motor and mechanical conditions, toxic and physical reactions, other inflammatory conditions and various neoplasms.

The increasing role of colonoscopy and polypectomy in the diagnosis and management of colorectal adenomas during the past decade has provided the histopathologist with an abundance of valuable material for study. This material is 
useful because, in most patients subjected to colonoscopy, the entire colorectum has been visualised at one examination and all the polyps removed for histological examination. Colonoscopies biopsies are now performed not only for the diagnosis of disease but also for the monitoring of the course of a wide variety of conditions and for the early detection of complications.

Colonoscopic biopsies are now performed not only for the diagnosis of disease but also for the monitoring of the course of a wide variety of conditions and for the early detection of complications. The colon and rectum can be sites for infections, idiopathic inflammatory bowel disease (IBD), vascular disorders, motor and mechanical conditions, toxic and physical reactions, other inflammatory conditions and various neoplasms. This material is useful because, in most patients subjected to colonoscopy, the entire colorectum has been visualised at one examination and all the polyps removed for histological examination.

\section{Materials and Methods}

This is a prospective study carried out over a period of 26 months from August 2013 to September 2015. The colonoscopic biopsy specimens were received to the Department of Pathology. All the cases irrespective of age and sex who presented to the Department of Gastroenterology and Surgery with various gastrointestinal symptoms who were subjected to colonoscopy for colorectal pathology were taken to study. All the colonoscopic biopsies received in Dept of Pathology, were considered in the study. The resected specimens of large intestine are excluded from the study.

Relevant clinical data was collected from patients through questionnaire, clinical examination, requisition forms and from case sheets. All colonoscopic biopsies received in Dept of Pathology were subjected to histopathological examination. The biopsy material, after 24 hours of fixation in $10 \%$ formalin is processed for paraffin embedding. Sections are cut at 5-6 mm thickness, cleared in xylene, hydrated and dehydrated in graded alcohols and stained with haematoxylin \& eosin stain. Special stains like PAS, Alcian Blue and Congo red are carried out wherever required along with routine haematoxylin and eosin staining. The clinical details of the cases like age, clinical history, clinical diagnosis was collected from the patients requisition forms. The histopathological findings were noted and the sections were submitted for special stains and results were noted accordingly.

\section{Results}

The present study was conducted from August 2013 to September 2015 in the Department of pathology. A total of 76 biopsies were studied. Among them 24 cases were non-neoplastic, 52 cases were neoplastic. Out of all neoplastic 19 were benign and 33 were malignant neoplasms. The present study involved 76 cases and colonoscopic biopsy was performed on patients of all age groups, ranging from a 5year old male child to an 81 years old female. The majority of cases were males $45(59.2 \%)$ and females 31 (40.8\%). [Table 1]

Table 1: Sex distribution of all colorectal lesions

\begin{tabular}{|l|c|c|}
\hline Sex & Number of cases & Percentage \\
\hline Male & 45 & $59.2 \%$ \\
\hline Female & 31 & $40.8 \%$ \\
\hline Total & 76 & $100 \%$ \\
\hline
\end{tabular}

Maximum numbers of cases were observed in the age group of $40-49$ years $(23.7 \%)$, followed by $30-39$ years $(22.4 \%)$ and $50-59$ years $(19.7 \%)$ and minimum number of cases were seen in the age group $80-89$ years $(1.3 \%)$. The mean age of the study was 42.89 .

The clinical features observed in order of frequency were rectal bleeding (altered and/or fresh) (86\%), constipation (61\%), weakness (59\%), pain abdomen $(43 \%)$ and diarrhoea $(33 \%)$. [Table 2] 
Table - 2: Clinical features of all cases

\begin{tabular}{|l|c|c|c|}
\hline Sl No & Clinical feature & $\begin{array}{c}\text { Number } \\
\text { of cases }\end{array}$ & Percentage \\
\hline 1. & Bleeding P/R & 65 & $86 \%$ \\
\hline 2. & Constipation & 46 & $61 \%$ \\
\hline 3. & Weakness & 45 & $59 \%$ \\
\hline 4. & Pain abdomen & 33 & $43 \%$ \\
\hline 5. & Diarrhea & 25 & $33 \%$ \\
\hline
\end{tabular}

In the present study of 76 colorectal biopsies: 24 cases were diagnosed as Non-neoplastic lesions, 19 cases were diagnosed as benign neoplasms and 33 cases were diagnosed as malignant neoplasms. [Table 3]

Table - 3: Distribution of all lesions

\begin{tabular}{|l|c|c|c|}
\hline Sl.no & Diagnosis & $\begin{array}{c}\text { Number of } \\
\text { cases }\end{array}$ & Percentage \\
\hline 1 & Non-neoplastic & 24 & $31.5 \%$ \\
\hline 3 & $\begin{array}{c}\text { Neoplastic- } \\
\text { benign }\end{array}$ & 19 & $25 \%$ \\
\hline & $\begin{array}{c}\text { Neoplastic- } \\
\text { malignant }\end{array}$ & 33 & $43.4 \%$ \\
\hline
\end{tabular}

In the present study, the range of age distribution of all non-neoplastic lesions was 5years to 62 years. There was clustering of cases between 2059 years, with maximum cases seen in 30-39 years. Mean age of non-neoplastic lesions was 34.91. There were 24 cases of benign neoplasms, out of which 15 cases were seen in males $(62.5 \%)$ and 9 cases were seen in females (37.5\%). Male: female ratio was 1.7:1.

In the present study of 76 colonoscopic biopsies, 24 cases were non-neoplastic lesions, out of these 6 cases were of Ulcerative colitis, 4 cases were of Tuberculous colitis, 5 cases were of Crohn's colitis, 3 cases were of chronic non-specific colitis, 2 cases of Solitary rectal ulcer, 2 cases of Amyloidosis and 1 case each of Acute colitis and Eosinophilic colitis.

Out of 19 benign neoplasms, 7 cases were of Hyperplastic polyps, 3 cases were of Juvenile polyps, 3 cases were of Tubular adenoma, 3 cases were of villous adenoma, there were 2 cases of Tubulovillous adenoma and 1 case of Lipoma. Majority of cases were seen in 40-49 years $(26.3 \%)$ age group followed by $3^{\text {rd }}, 4^{\text {th }}$ and $6^{\text {th }}$ decades $(15.7 \%$ each). Mean age of benign neoplasms was 31.36 .

In the present study, malignant neoplastic lesions were distributed between $20-89$ years with clustering of cases seen between $30-79$ years, with predominance in $6^{\text {th }}$ decade. Mean age of malignant neoplasms was 55.36. Rectum was the commonest site in large intestinal tumours with 10 (30.3\%) cases followed by ascending colon with 6 cases $(18.2 \%)$.

The predominant histological variant among all colorectal malignancies is Adenocarcinoma with 27 cases $(81.8 \%)$. 4 cases $(12.1 \%)$ of Mucinous adenocarcinomas were diagnosed. 1 case of signet-ring adenocarcinoma was diagnosed $(3.03 \%)$ and characterised by tumour cells arranged in sheets in lamina propria and infiltrating the sub-mucosa. The tumour cells had abundant intracellular mucin which was PAS positive with eccentric hyperchromatic nuclei. Out of 27 cases of Adenocarcinoma, 11 cases were graded as well differentiated adenocarcinoma $(40.7 \%)$ and were characterised by malignant glands invading the sub-mucosa. The glands were well formed and lined by tallcolumnar cells with hyperchromatic nuclei and only mild nuclear stratification [Table 4]

Table 4: Histological types of malignant colorectal neoplasms

\begin{tabular}{|l|c|c|}
\hline Microscopic appearance & $\begin{array}{c}\text { Number } \\
\text { of cases }\end{array}$ & Percentage \\
\hline Adenocarcinoma & 27 & $81.8 \%$ \\
\hline Mucinous adenocarcinoma & 4 & $12.1 \%$ \\
\hline Signet ring cell carcinoma & 1 & $3.03 \%$ \\
\hline GIST & 1 & $3.03 \%$ \\
\hline Total & 33 & $100 \%$ \\
\hline
\end{tabular}

Majority i.e., 14 cases (51.9\%) of the adenocarcinomas were moderately differentiated which showed malignant glands with irregular outline. There was loss of nuclear polarity and variation in nuclear size and shape. There were 2 cases of poorly differentiated adenocarcinomas and they displayed highly irregular or ill-formed tubular structures. The tumour cells were seen in chords, clumps or sheets and had pleomorphic hyperchromatic nuclei with prominent nucleoli 


\section{JMSCR Vol||07||Issue||11||Page 517-523||November}

and scanty cytoplasm. The tumour cells were PAS positive.

Ulcerative colitis (Transverse colon)Inflammatory infiltrate in lamina propria, crypt distortion \& crypt abscess formation (H\&E;10x)

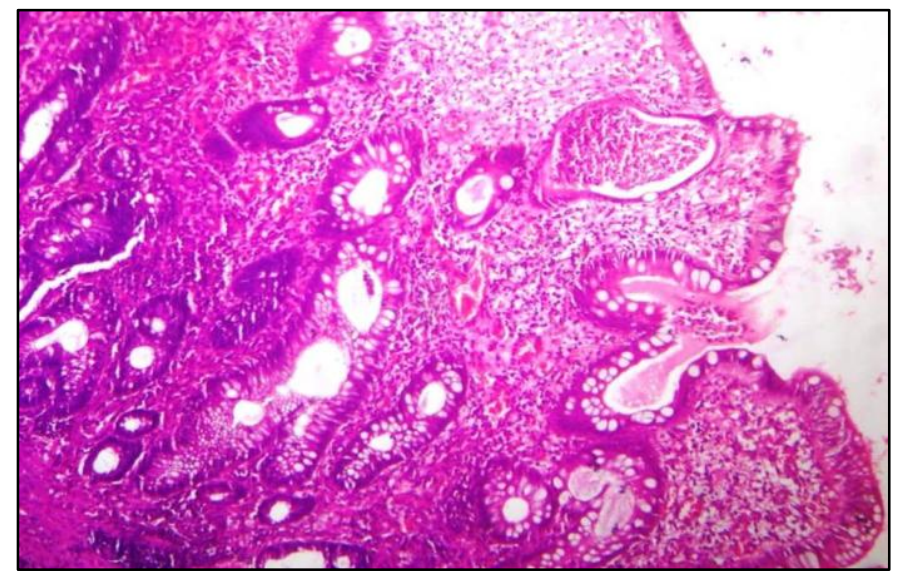

Tuberculous colitis- large confluent granulomas, caseating necrosis $(H \& E ; 40 x)$

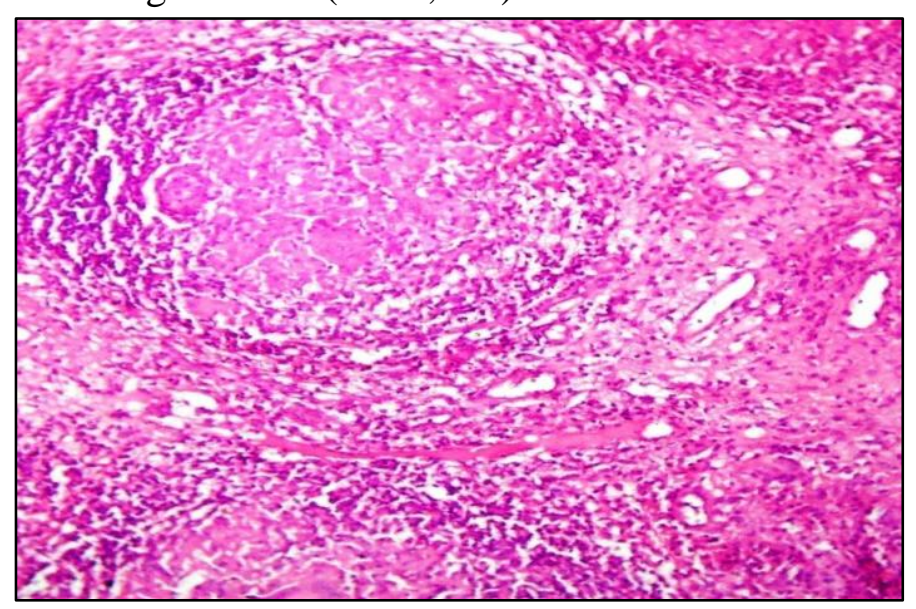

Hyperplastic polyp (Sigmoid colon)- showing tufting of epithelium composed predominantly of goblet cells $(H \& E ; 10 x)$

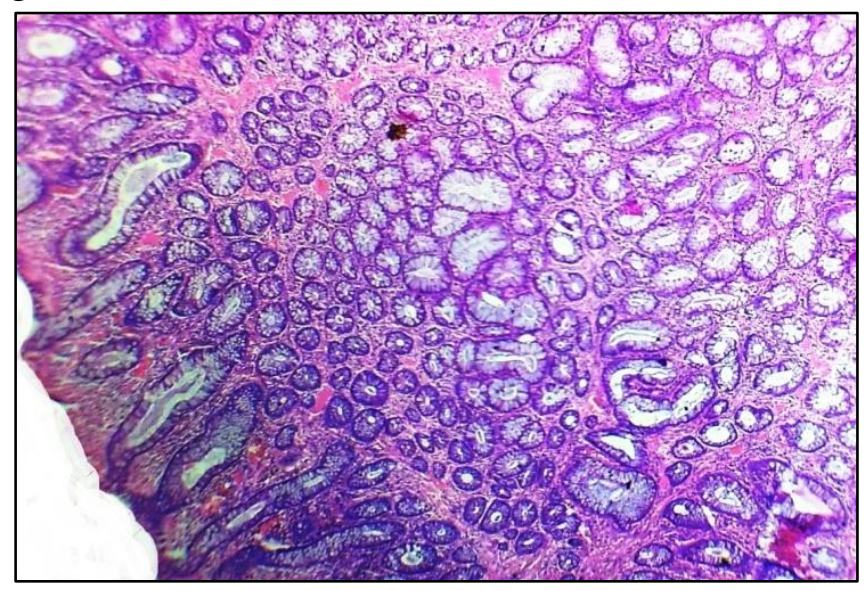

Tubulovillous adenoma (Ascending colon) admixture of villous \& glandular structures $(\mathrm{H} \& \mathrm{E} ; 10 \mathrm{x})$

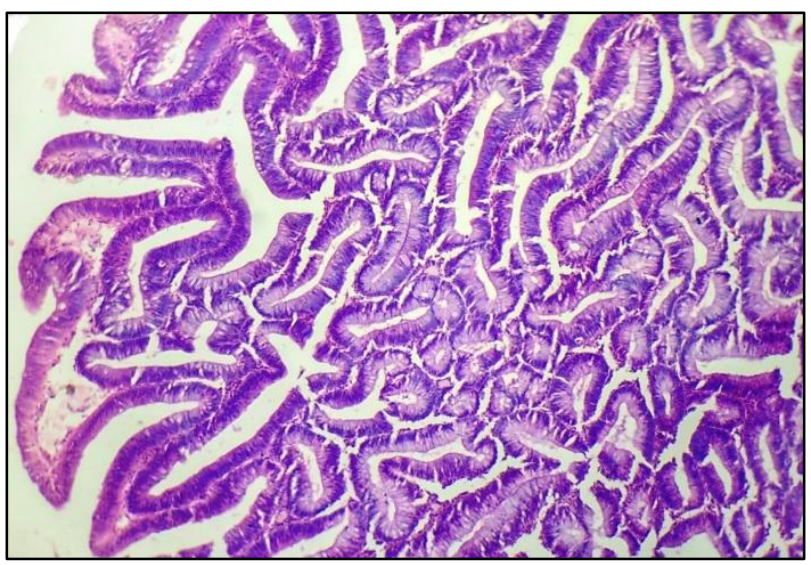

Well-differentiated adenocarcinoma (Descending colon) with $>95 \%$ glandular elements (H\&E;10x)

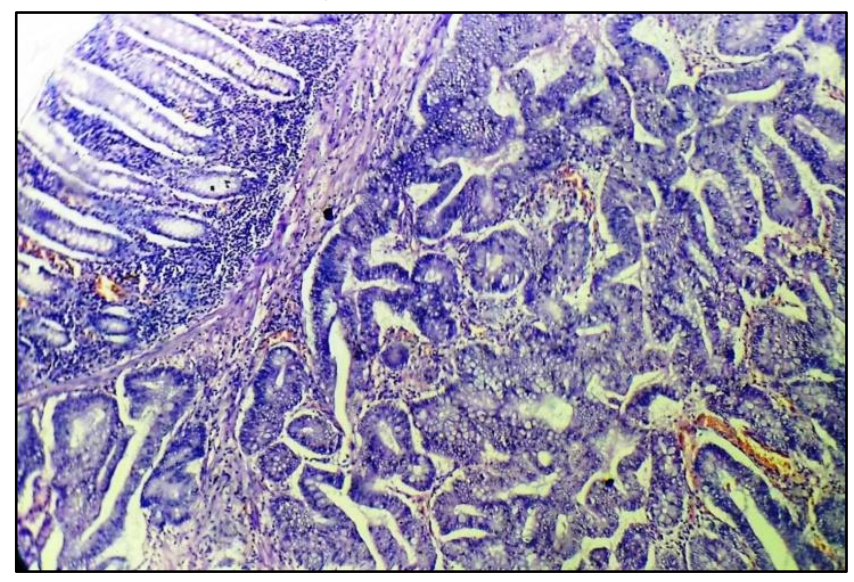

Signet ring cell carcinoma (Ascending colon)showing signet ring cells with prominent intracytoplasmic mucin with nucleus pushed to periphery (H\&E; 40x)

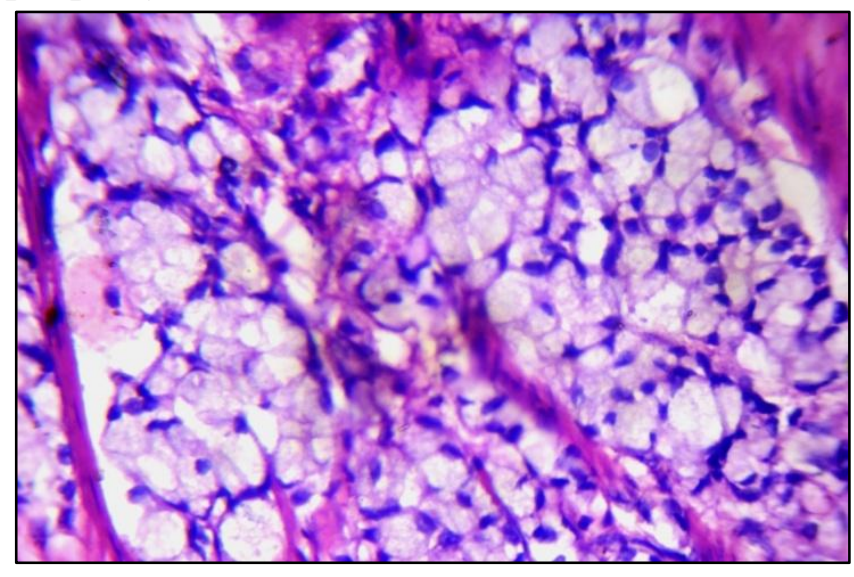

\section{Discussion}

Intestinal tumours display a wide spectrum of gross and histological variations. Colonic 
conditions like infections, IBD, polyps and colorectal tumours are important lesions which often require colonic biopsy for their conclusive diagnosis.

A rising trend in the incidence and prevalence of inflammatory bowel disease (IBD) in Asia has been recognized for the past two decades. The highest incidence rates are recorded from India, Japan and the Middle East and there exists a genetic predisposition of South Asians (Indians, Pakistanis and Bangladeshis) to ulcerative colitis (UC) ${ }^{(2)}$.

Colorectal cancer (CRC) is a formidable health problem worldwide. As per the GLOBOCAN project undertaken by WHO in 2008, It is the third most common cancer in men (663000 cases, $10.0 \%$ of all cancer cases) and the second most common in women (571000 cases, $9.4 \%$ of all cancer cases $)^{(3)}$. Worldwide, an estimated 1.2 million cases of colorectal cancer occurred in $2008^{(3)}$. About 608,700 deaths from colorectal cancer occurred in 2008 worldwide, accounting for $8 \%$ of all cancer deaths ${ }^{(3)}$.

In India, the annual incidence rates (AARs) for colon cancer and rectal cancer in men are 4.4 and 4.1 per 100000 , respectively. The AAR for colon cancer in women is 3.9 per $100000^{(4)}$. In the 2013 report, the highest AAR in men for CRCs was recorded in Thiruvananthapuram (4.1) followed by Bangalore (3.9) and Mumbai (3.7). The highest AAR in women for CRCs was recorded in Nagaland (5.2) followed by Aizwal (4.5) ${ }^{(4)}$

The current recommendation for colorectal cancer screening in most countries is to begin screening at age 50 for men and women who are at average risk for developing colorectal cancer. While colonoscopy is a highly sensitive test, it requires a skilled examiner, involves greater cost, and is less convenient and has more risk for the patient ${ }^{(5)}$.

in contrast to the study of R.H.Teague et al, Sidney. J et al and Azar Qayyam et al, where nonneoplastic lesions were dominant followed by neoplastic lesions, in our study, out of 76 cases, $24(31.5 \%)$ cases were non-neoplastic and 33 $(43.4 \%)$ cases were neoplastic malignant. Benign neoplastic lesions were 19 accounting to $25 \%$, which matched with the study of R.H.Teague et al ${ }^{(1973)^{(6)}}$

Dickinson et $\mathrm{al}^{(7)}$ in their study of 74 cases described 15 cases (20.3\%) of Acute nonspecific colitis.

Tablot and price ${ }^{(8)}$ described acute non-specific inflammation of the colon as displaying focal polymorphic exudate in the lamina propria, infiltrating crypts and surface epithelium with an arbitrary involvement of 3-4 crypts, not necessarily contagiously; accompanied by mild increase in lymphocytes and plasma cells; straight crypts and mild decrease to a normal number of goblet cells.

In the present study, there was one case of Acute colitis $(4.2 \%)$, characterised by neutrophilic and mononuclear cell infiltrate in the lamina propria. No crypt abscesses or branching or decrease in goblet cells were observed.

Dickinson et al described 20 cases (27\%) of chronic colitis in their study of 74 cases.

Talbot and Price ${ }^{(8)}$, Carpenter and Tally in $2000^{(9)}$, diagnosed cases showing a marked increase in plasma cells and lymphocytes in the lamina propria, focal or diffuse with intact and well-aligned crypts with/without some goblet cell depletion as 'chronic non-specific inflammation of the colon'.

The present study showed 3 cases of chronic nonspecific colitis with predominantly lymphoplasmacytic infiltrate in the lamina propria with well-preserved mucosal glands and normal goblet cells.

The present study was compared with the study series of Hassan Abdulla Al-aquli et al ${ }^{(10)}$. Of the 31 cases $(11.75 \%)$ which included $21(67.7 \%)$ were of Juvenile polyps, 6 cases (19.3\%) were of Hyperplastic polyps and 4 cases $(13 \%)$ were of Hamartomatous polyps. In the present study Hyperplastic polyps were predominant, followed by Juvenile polyps.

The age range in the present study was $28-81$ years, with a mean age of 55.36 years. This was consistent with study conducted by Fazeli MS et 
al $^{(11)}$.

In the present study, the incidence of colorectal cancers was more in males than in females, with a male: female ratio of 1.2:1. This finding was consistent with sex incidence in the studies done by Mostafa $\mathrm{G}$ et al and Newland $\mathrm{RC}$ et $\mathrm{al}^{(12)}$.

Most colorectal carcinomas are located in the sigmoid colon and rectum, but there is evidence of changing distribution in recent years, with an increasing proportion of more proximal carcinomas (13)

In high-risk countries, colorectal carcinoma most commonly arises in the rectosigmoid region, but though we are in low risk countries we encountered more lesions in the distal region whether it was due to environmental factors, or easy approachability detected them at early stage, as many of these were well differentiated adenocarcinomas.

\section{Conclusion}

A variety of both Non-neoplastic and Neoplastic lesions were reported in the present study across a wide age distribution and the findings correlated well with that of similar studies. Much difficulty was encountered in correct interpretation, because of the small size of the biopsy specimens received. A greater awareness of the disease and understanding of pathogenesis on the part of the pathologist was felt to be necessary for a better improved diagnosis.

Histopathological diagnosis correlated well with the colonoscopic diagnosis offered especially in malignant neoplasms. Therefore, colonoscopic biopsy is an important tool in the diagnosis of large bowel diseases. The histopathological study of colonoscopic biopsy specimens should be comprehensive by constant correlation with the clinical details and colonoscopic features. Colonoscopic examination with biopsy may be used for follow up of Inflammatory bowel diseases and to define epithelial dysplasia in cases of chronic colitis and thus help in the early detection of carcinoma in such cases.
Hence, colonoscopic biopsy has improved the role of pathologists in the diagnosis and management of large bowel diseases while underscoring a constant correlation with clinicians, but a correct interpretation still proves to be an exciting diagnostic challenge for pathologists because of small size of the specimens.

\section{References}

1. Ferlay J, Shin HR, Bray F, Forman D, Mathers C, Parkin DM. Estimates of worldwide burden of cancer in 2008: GLOBOCAN 2008. Int J Cancer.2010; 127:2893-917.

2. Vineet AHUJA and Rakesh K TANDON ; Inflammatory bowel disease in the AsiaPacific area: A comparison with developed countries and regional differences, 2010: Gastroenterology and Hepatology, Journal of digestive diseases, Vol 11, Issue 3, 1751-2980.

3. GLOBOCAN 2008 (http://globocan.iarc.fr/factsheets/cancers/c olorectal.asp) (2008).

4. NCRP (2013) Three-year report of the population based cancer registries- 20092011. National cancer registry programme, Indian council of medical research (ICMR), Bangalore, India, 2013.

5. Winawer SJ. The multidisciplinary management of gastrointestinal cancer. Colorectal cancer screening. Best Pract Res Clin Gastroenterol. 2007;21(6):10311048

6. Teague RH, Read AE. Polyposis in ulcerative colitis. Gut 1975;16:792.

7. Dickinson RJ, Gilmour HM, Mc Clelland DBL. 1979. "Rectal biopsy in patients presenting to an infectious unit with diarrhoea disease". Gut, 20:141.

8. Tablot C, Price AB. 1987. Biosy Pathology in Colorectal Disease. University Press, Cambridge.

9. Carpenter HA, Talley NJ. 2000. "The importance of clinicopathological 
correlation in the diagnosis inflammatory diseases of the colon: histological patterns with clinical implications". Am J Gastroenterol, 95:878

10. Hassan Abdulla AL-aquili. Clinical, colonoscopic and histopathological evaluation of lower gastrointestinal bleeding. Kufa Med. Journal 2012. Vol .15. no. 1

11. Fazeli MS, Adel MG, Lebaschi AH. Colorectal carcinoma: A retrospective, Descriptive study of Age, Gender, subsite, stage and differentiation in Iran from 1995 to 2001 as observed in Tehran University. Dis Colon Rectum. 2007;50:990-995

12. Newland RC, Chapuis PH, Smyth EJ. The prognostic value of substaging colorectal carcinoma. A prospective study of 1117 cases with standardized pathology. Cancer 1987;60:852-857

13. Hamilton S.R., Aaltonen L.A. (Eds.): World Health Organization Classification of Tumours. Pathology and Genetics of Tumours of the Digestive system. IARC press: Lyon 2000. 